\title{
Étude de l'amélioration des propriétés des anodes par le brai modifié
}

Julie Bureau, Duygu Kocaefe*, Yasar Kocaefe et Dipankar Bhattacharyay

Chaire institutionnelle sur les matériaux industriels (CHIMI) et Centre de recherche sur l'Aluminium-REGAL

Université du Québec à Chicoutimi, 555 boulevard de l'Université, Chicoutimi, Québec, G7H 2B1, Canada

Mots clés : Production d’aluminium, Propriétés d’anodes, Additifs chimiques, Brais modifiés

\section{RÉSUMÉ}

Dans l'industrie de l'aluminium, les anodes sont utilisées afin de fournir le carbone nécessaire à la réduction électrolytique de l'aluminium. Toutefois, la compatibilité des matériaux de base est compromise par la qualité décroissante du coke et du brai nuisant à l'obtention des propriétés finales des anodes. L’objectif de ce travail est de vérifier si l'amélioration de la mouillabilité du coke par un brai modifié chimiquement peut contribuer à améliorer certaines des propriétés d'anodes. La méthodologie prévoit la fabrication d'anodes au laboratoire de carbone de l'UQAC à partir de brais modifiés à l'aide d'un additif chimique et la comparaison avec des anodes standards. Deux additifs chimiques ont été testés. Les anodes sont ensuite carottées et les échantillons sont caractérisés à l'état cru et cuit selon les normes appropriées. Dans cette étude, les deux additifs sélectionnés ont permis de démontrer une amélioration significative de certaines des propriétés des anodes.

\section{INTRODUCTION}

Dans le but d'obtenir l'aluminium primaire en fusion, l'alumine $\left(\mathrm{Al}_{2} \mathrm{O}_{3}\right)$ dissoute dans la cryolithe (le bain) est réduite grâce au procédé électrolytique élaboré par Hall-Héroult selon l'Équation globale $1:^{[1,2]}$

$$
2 \mathrm{Al}_{2} \mathrm{O}_{3} \text { (bain) }+3 \mathrm{C} \text { (anode) } \rightarrow 4 \mathrm{Al}_{(\text {liq) }}+3 \mathrm{CO}_{2} \text { (g) }
$$

Le carbone nécessaire à la réduction de l'aluminium, soit $0,334 \mathrm{~kg}$ de $\mathrm{C} / \mathrm{kg}$ d'Al, est fourni par les anodes. L'énergie nécessaire à la réaction est de 6,34 $\mathrm{kWh} / \mathrm{kg} \mathrm{Al} .{ }^{[1,2]}$ Cependant, ces données représentent la consommation minimale théorique et ne tiennent pas compte des diverses pertes selon l'efficacité du procédé. La consommation réelle est d'au moins $0,4 \mathrm{~kg}$ de C / kg Al et d'environ 13,0 kWh / kg Al et plus. ${ }^{[1,2]}$ Le courant nécessaire à l'électrolyse dans la plupart des usines est approximativement de 200 à $400 \mathrm{kA}$. De nouvelles technologies permettent actuellement l'usage d'un courant se situant entre 500 et $600 \mathrm{kA}^{[2]}$

La fabrication d'une anode crue comprend la préparation de la pâte d'anode qui se compose des agrégats secs (coke de pétrole calciné, mégot, rejets crus et cuits) ( $85 \%$ ) et d'un liant (brai de goudron de houille) ( $15 \%) \cdot{ }^{[1]}$ La cuisson des anodes entraîne la dévolatilisation des composants légers du brai et engendre la formation d'une matrice solide due à la carbonisation, ce qui 
contribue à l'obtention des propriétés finales des anodes cuites. ${ }^{[1-4]}$ La densité moyenne d'une anode cuite est d'environ $1,55 \mathrm{~kg} / \mathrm{dm}^{3}$ et possède une durée de vie d'environ 21 jours. ${ }^{[1]}$ Une anode de qualité se doit de résister à l'environnement hostile de la cellule électrolytique sans toutefois nuire à l'électrolyse. Parmi les caractéristiques essentielles d'une anode, celle-ci devrait avoir une haute densité et une faible perméabilité aussi bien qu’une faible porosité pour diminuer la consommation de carbone et augmenter le cycle de vie de l'anode. De plus, afin de minimiser la consommation excessive de carbone, une haute résistance à l’oxydation est requise. De fait, le carbone des anodes peut réagir en surface avec l'air (la réactivité à l'air) et libérer du dioxyde de carbone. En plus, le dioxyde de carbone libéré lors de l’électrolyse au bas de l'anode peut réagir avec le carbone de l'anode pour former du monoxyde de carbone (la réactivité au $\mathrm{CO}_{2}$ ). Enfin, de l’oxydation sélective peut survenir dû à la réactivité inégale des différentes particules de coke et la matrice liante, ce qui fragilise la structure. Par conséquent, des particules de cokes peuvent se libérer seules de la matrice ou encore être soustrait par des forces mécaniques, thermiques ou magnétiques. Ce phénomène est communément appelé poussiérage. Ces réactions secondaires augmentent la consommation de carbone. En outre, un grand degré de pureté de l'anode est nécessaire afin d’éviter la contamination de l'aluminium produit et la consommation prématurée de l'anode. De plus, une faible résistance électrique spécifique est requise afin de réduire la consommation énergétique. Toutefois, une faible résistance électrique implique une conductivité thermique élevée. Un accroissement de la conductivité thermique favorise l'augmentation de la température à la surface de l'anode et conséquemment l'augmentation de la réactivité à l'air. À l’inverse, une résistance électrique spécifique élevée signifie la présence possible de fissures de l'anode et une augmentation de la consommation énergétique. Une faible résistance électrique est donc préférable jusqu’à un certain seuil. Ensuite, une résistance élevée aux chocs thermiques est bénéfique dans le but de diminuer les risques de fissuration lorsque l’anode est introduite dans la cellule électrolytique chaude. Enfin, une résistance mécanique suffisante conserve son intégrité et favorise sa manipulation. ${ }^{[1,2,5]}$

Plusieurs auteurs rapportent différentes méthodes de modifications des propriétés du brai par l'ajout d'additifs. ${ }^{[7-14]}$ Toutefois, l'interaction entre le brai et le coke n'est pas toujours étudiée. Rocha et al. ont publiés plusieurs articles utilisant les tests de mouillabilité pour évaluer l'interaction coke/brai. ${ }^{[8,9,12,14]}$ Une seule de ses études emploie le brai de goudron de houille (BGH), qui est utilisé en général dans la production des anodes, comme type de brai. En effet, 
Rocha et al. proposent une modification du BGH par l'ajout d'additif dont la composition exacte n’est pas spécifiée. ${ }^{[8]}$ Les additifs nommés A et B renfermaient respectivement $22,8 \%$ et 10,8 \% d'oxygène. Les essais ont permis d'affirmer qu'un ajout de 0,025 g/g de l'additif A ne modifie pas les propriétés du brai. Par contre, l'ajout de 0,05 et 0,10 g/g de l'additif A diminuent la tension de surface et augmente la mouillabilité. Des résultats similaires sont obtenus pour l'additif B, mais nécessite un ajout minimal de 0,15 g/g. Enfin, peu de chercheurs ont publié des travaux portant sur l'ajout d'additifs chimique lors de la fabrication des anodes. En 1999, la Commission européenne a publié un rapport qui présente des modifications du BGH avec du carboxyméthyl-cellulose de sodium (CMCNa) 0,05 g/ml eau, du Mobilsol 40 (M40) ou des granules de polystyrène (PS). ${ }^{[13]}$ Les proportions suivantes d'additifs ont été ajoutées à la pâte d'anode lorsque placées dans le mélangeur, soit : $0,75 \%$ en masse sèche de CMCNa; 0,01; 0,03 et 0,05 g/g de M40; 0,05 g/g de PS. L'ajout de CMCNa à la pâte d'anode produit des anodes de pauvre qualité. Les chercheurs ont établi que le CMCNa a encapsulé le BGH nuisant à la pénétration du coke. L'ajout de Mobilsol 40 ou de PS à $100{ }^{\circ} \mathrm{C}$ ou encore $180-220{ }^{\circ} \mathrm{C}$ n’améliorent pas de façon significative l'ensemble des propriétés des anodes.

L'objectif de cette étude est de produire des anodes de bonne qualité à partir des matières premières offertes sur le marché. Plus spécifiquement, le but de cette étude est d'explorer s’il est possible d'améliorer certaines propriétés des anodes par l'ajout d'additifs chimiques dans le brai. En outre, les additifs choisis doivent contenir des groupements fonctionnels susceptibles de se lier chimiquement au brai tout en étant peu coûteux. De plus, leurs compositions chimiques doivent favoriser la compatibilité entre le coke et le brai en se liant également au coke. Afin de permettre la décomposition des réactifs et contribuer à la valeur en cokéfaction, le point d'ébullition des additifs doit être inférieur à la température finale au cours de l'étape de cuisson des anodes. Enfin, dans le but de prévenir la contamination des anodes et maintenir l'efficacité du procédé d'électrolyse, les additifs chimiques sélectionnés de même que leurs produits de décomposition ne doivent pas contenir d’atome jugé nuisible.

Une étude par Bureau et al. a permis d'identifier des additifs chimiques prometteurs pour la fabrication des anodes. ${ }^{[15]}$ Selon ces résultats obtenus, des anodes de laboratoire ont été fabriquées et caractérisées dans cette étude afin de déterminer l'effet de l'ajout d'un brai modifié sur les propriétés des anodes comparativement à une anode standard. 


\section{MATÉRIEL ET MÉTHODE}

\section{Matériel}

Dans cette étude, des anodes ont été produites au laboratoire de carbone de l'UQAC. Les anodes standards qui y sont fabriquées possèdent des propriétés similaires à celles des anodes industrielles. Afin de fabriquer ces anodes, deux brais de goudron de houille (brai B1 et brai B4) provenant de l'industrie de l'aluminium ont été employés tels que reçus. Ces brais ont été sélectionnés en fonction des résultats d'une étude antérieure sur les brais B1, B2, B3 et B4. ${ }^{[15]}$ Dans la même étude, plusieurs additifs ont été testés; et deux de ces additifs sont retenus pour le travail présenté dans cet article. ${ }^{[15]}$ Donc, les brais sélectionnés (B1 et B4) ont été modifiés avec un tensioactif (additif A1) ou un agent de modification de surface (additif A3), tous les deux achetés chez Alfa Aesar. Afin de modifier un brai, un additif chimique renfermant des groupements fonctionnels pouvant enrichir l’interaction avec un coke standard a été ajouté dans le processus de fabrication des anodes. Les propriétés des brais B1 et B4 ont été améliorées en raison de la réaction chimique avec l'additif ajouté qui à son tour a amélioré l'interaction cokebrai, c'est-à-dire la mouillabilité. La modification des spectres FTIR indique qu'une réaction chimique a bien eu lieu. Les additifs sélectionnés ont été identifiés comme étant peu coûteux, avec un point d'ébullition inférieur à la température maximale de cuisson et dont les produits de décomposition ne seront pas nuisibles au processus de fabrication de l'aluminium étant donné la nature de leurs compositions chimiques.

Pour la modification des brais, une concentration (ci) en pourcentage de la masse d'additif sur la masse de l'échantillon, soit $0,005<\mathrm{c} 1<\mathrm{c} 3<\mathrm{c} 4 \leq 0,05 \mathrm{~g} / \mathrm{g}$, ont été employés. Ensuite, un coke de pétrole standard (Coke 1) utilisé dans l’industrie de l'aluminium a été employé afin de fabriquer des anodes de laboratoire. Enfin, les autres agrégats secs nécessaires à la fabrication des anodes, soit le mégot et les rejets d'anodes (crus et cuits) qui sont aussi similaires à ceux utilisés dans l'industrie de l'aluminium, ont été ajoutés. Chacune des matières premières utilisées provient du même lot afin de diminuer l’impact des impuretés présentes sur les résultats obtenus. Les propriétés des matières premières sont présentées dans le Tableau 1 pour les brais, le Tableau 2 pour le coke et le Tableau 3 pour les additifs. Le Tableau 4 présente un sommaire des travaux présentés dans l'étude antérieure mentionnée ci-haut. ${ }^{[15]}$ À noter qu’habituellement, les résultats de mouillabilité sont présentés en comparant les angles de mouillage. Toutefois, les tests de mouillabilité ne sont pas réalisés sur une surface complètement solide. Afin d’approximer une 
surface lisse, de petites particules de cokes ont été compactées pour former le lit de coke. Étant donné la nature de la surface, les résultats des tests goutte-sessile sont affectés par deux phénomènes, soit: le mouillage et la pénétration. De plus, ces deux phénomènes sont également liés, car si le liquide ne mouille pas le solide, il ne pénètre pas dans le lit de coke. Par conséquent, les différentes expérimentations ont été comparées selon le temps de pénétration, car un meilleur mouillage entraînera des temps de pénétration plus courts.

Tableau 1. Propriétés des brais non modifié

\begin{tabular}{|l|c|c|}
\hline \multirow{2}{*}{ Propriétés } & \multicolumn{2}{|c|}{ Brai de goudron de houille } \\
\cline { 2 - 3 } & $\mathrm{B} 1$ & $\mathrm{~B} 4$ \\
\hline Cendre à $900{ }^{\circ} \mathrm{C}(\mathrm{g} / \mathrm{g})$ & 0,0017 & 0,0012 \\
\hline$\beta$ Résine $(\mathrm{g} / \mathrm{g})$ & 0,221 & 0,222 \\
\hline Densité $(\mathrm{g} / \mathrm{ml})$ & $1,312\left(\right.$ à $\left.25^{\circ} \mathrm{C}\right)$ & $1,320\left(\mathrm{à} 20^{\circ} \mathrm{C}\right)$ \\
\hline Insoluble dans la quinoléine $(\%)$ & 7,5 & 6,9 \\
\hline Insoluble dans le toluène $(\%)$ & 29,6 & 29,1 \\
\hline Valeur de cokéfaction $(\%)$ & 59,9 & 59,1 \\
\hline Point de ramollissement $\left({ }^{\circ} \mathrm{C}\right)$ & 121,5 & 119,6 \\
\hline Viscosité dynamique à $170^{\circ} \mathrm{C}(\mathrm{mPa} \cdot \mathrm{s})$ & 2070 & 1390 \\
\hline
\end{tabular}

Tableau 2. Propriétés du coke

\begin{tabular}{|l|c|}
\hline Propriétés & Coke de pétrole frais (Coke 1) \\
\hline Densité en vrac tassée $\left(\mathrm{g} / \mathrm{m}^{3}\right)$ & 0,89 \\
\hline Densité réelle $\left(\mathrm{g} / \mathrm{m}^{3}\right)$ & 2,06 \\
\hline Réactivité au $\mathrm{CO}_{2}(\%)$ & 9 \\
\hline Contenu en cendre (\%) & 0,2 \\
\hline Teneur en humidité $(\%)$ & $<0,1$ \\
\hline $\mathrm{Na}(\mathrm{ppm})$ & 70 \\
\hline $\mathrm{Si}(\mathrm{ppm})$ & 100 \\
\hline $\mathrm{P}(\mathrm{ppm})$ & 6 \\
\hline $\mathrm{S}(\%)$ & 2,75 \\
\hline $\mathrm{Ca}(\mathrm{ppm})$ & 100 \\
\hline $\mathrm{V}(\mathrm{ppm})$ & 310 \\
\hline $\mathrm{Fe}(\mathrm{ppm})$ & 200 \\
\hline $\mathrm{Ni}(\mathrm{ppm})$ & 200 \\
\hline
\end{tabular}


Tableau 3. Propriétés des additifs ${ }^{[15]}$

\begin{tabular}{|c|c|c|c|c|}
\hline Additif & $\begin{array}{c}\text { Apparence physique à } \\
\text { température ambiante }\end{array}$ & $\begin{array}{c}\text { Point de fusion } \\
(\mathrm{PF}),{ }^{\circ} \mathrm{C}\end{array}$ & $\begin{array}{c}\text { Point d'ébullition } \\
(\mathrm{PE}),{ }^{\circ} \mathrm{C}\end{array}$ & Classe générique \\
\hline A1 & Solide & 58 & 238 & $\begin{array}{c}\text { Ester d'acide } \\
\text { carboxylique } \\
\text { aliphatique-poly-ol et } \\
\text { longue chaîne } \\
\text { aliphatique }\end{array}$ \\
\hline A3 & Liquide & 7.5 & 248 & Phényl-alkyl-aldéhyde \\
\hline
\end{tabular}

Tableau 4. Sommaire des travaux présentés dans l'étude antérieure ${ }^{[15]}$

\begin{tabular}{|c|c|c|c|}
\hline $\begin{array}{c}\text { Brai } \\
\text { utilisé }\end{array}$ & $\begin{array}{c}\text { Temps de } \\
\text { mouillage } \\
\text { total (s) }\end{array}$ & $\begin{array}{l}\text { Sommaire de l'analyse des } \\
\text { résultats FTIR }\end{array}$ & Mécanisme possible \\
\hline B1 & 168,3 & S.O.** & S.O.** \\
\hline B4 & 139,1 & S.O.** & S.O.** \\
\hline A1c1B1* & 140,6 & L'additif A1 modifie & La présence de ses chaînes \\
\hline A1c3B1* & 56,1 & chimiquement le brai en & aliphatiques courtes peut favoriser les \\
\hline A1c2B4* & 69,3 & $\begin{array}{c}\text { augmentant le nombre de } \\
\text { chaînes aliphatiques présentes } \\
\text { par rapport au nombre des } \\
\text { composés aromatiques. }\end{array}$ & $\begin{array}{l}\text { réactions de condensation et de } \\
\text { cyclisation intramoléculaire favorable } \\
\text { à une augmentation de la valeur en } \\
\text { cokéfaction. }\end{array}$ \\
\hline A3c2B1* & 169,1 & \multirow{4}{*}{$\begin{array}{l}\text { L’additif A3 transforme } \\
\text { chimiquement le brai en } \\
\text { augmentant la présence } \\
\text { d’hétéroatomes. }\end{array}$} & \multirow{4}{*}{$\begin{array}{c}\text { L'augmentation d'hétéroatomes } \\
\text { signifie une augmentation possible du } \\
\text { nombre de liaisons hydrogènes, } \\
\text { d'interactions électrostatiques, } \\
\text { d'interactions acide-base et de liaisons } \\
\text { covalentes entre le brai et le coke. }\end{array}$} \\
\hline A3c4B1* & 97,2 & & \\
\hline A3c2B4* & 117,8 & & \\
\hline A3c4B4* & 68,1 & & \\
\hline
\end{tabular}

* Brai modifié (Exemple: A3c2B1 signifie que l'additif A3 est ajouté selon une concentration c2 pour modifier le brai B1).

** S.O.: sans objet.

\section{Préparation des matières premières}

Afin de fabriquer des anodes, la matière première doit être préparée. Les agrégats secs sont d'abord tamisés afin de séparer les différentes fractions. Des quantités différentes pour chacune 
des granulométries des agrégats secs sont pesées selon une recette prédéterminée. Les agrégats secs sont ensuite bien mélangés et chauffés avant de les ajouter au malaxeur. Pendant ce temps, le brai non modifié est chauffé dans un four et est ajouté directement au coke préalablement placé dans le malaxeur. Pour les brais modifiés, deux méthodologies ont été expérimentées. La première méthode prévoit d'ajouter l'additif directement au brai non modifié quelques instants avant la fabrication de l'anode. Le brai non modifié est préliminairement chauffé dans un four afin d'atteindre une température cible au-dessus du point de ramollissement. Lorsque les températures du brai, du coke et du moule sont adéquates pour effectuer le mélange, l’additif est ajouté au brai. Une brève agitation est effectuée et le mélange de brai est déversé dans la cuve pour être mélangé au coke. La seconde méthode consiste à préalablement modifier le brai avec l'additif chimique dans une enceinte sous flux d'azote. Le brai non modifié est chauffé avec un pistolet thermique (Master Appliance corp. Model VT-750C) afin d'obtenir une pâte visqueuse dont la température se situe au-dessus du point de ramollissement. La température interne du brai est mesurée à l'aide d'un thermocouple relié à un thermomètre électronique (Fluke 52 II). Puis, l'additif est ajouté au brai et agité jusqu’à l'obtention d'un mélange uniforme. Le brai modifié est refroidi graduellement sous le point de ramollissement, toujours sous flux d'azote. Le brai modifié est ensuite récupéré, broyé grossièrement et scellé afin de le conserver jusqu’à l'étape de préchauffage lors de la fabrication de l’anode.

\section{Fabrication des anodes}

La fabrication des anodes s'effectue selon la méthodologie développée à l'UQAC. Dans cette étude, la recette d’agrégat sec, les températures et le temps de préchauffage, les paramètres des procédés de malaxage et de vibrocompaction sont tous maintenus constants. Le seul paramètre modifié se situe par rapport au brai tel qu'expliquer dans la section précédente. Deux brais non modifiés (brais B1 et B4) ont été employés et deux méthodes de modification du brai ont été expérimentées. Ces deux brais ont été choisis puisque les brais B1 et B4 possèdent l'interactivité la plus faible et la plus forte avec le coke, respectivement. ${ }^{[15]}$ Conséquemment, une amélioration des propriétés d'anodes fabriquées à partir du brai B1 et B4 implique une possible amélioration pour les brais intermédiaires.

Une première série d’anodes sont fabriquées à partir du brai non modifié ayant démontré la plus faible compatibilité comparativement aux autres brais non modifiés, soit le brai B1. D’abord, une 
anode standard est produite à partir du brai B1 non modifié et du Coke 1 comme base de référence (anode 1). Puis deux anodes, de même concentration d’additif, sont fabriquées afin de choisir la méthode de modification appropriée selon la méthodologie de fabrication des anodes à l'UQAC (anodes 2 et 3). L'additif A1 obtient une amélioration de la mouillabilité plus importante comparativement à l’additif A3. L’additif A1 est donc sélectionné afin de déterminer l'impact d'autres modifications (type et concentration de l'additif) pour les anodes suivantes. Une concentration c3 a été choisie pour modifier le brai B1 avec l'additif A1 puisque le temps de mouillage total de ce brai, nommé A1c3B1, est similaire au temps de mixage des agrégats secs et du brai en milieu industriel (Tableau 4). Ensuite, l'effet de la concentration de l'additif A1 présent est étudié en employant une concentration moindre de c1 (anode 4). Enfin, l'effet de l'ajout de l'additif A3 pour une concentration c4 est observé par la fabrication de l'anode 5.

Puis, selon la quantité des matériaux encore disponibles, une deuxième série d'anodes sont produites avec le brai modifié ayant démontré la plus grande amélioration de la mouillabilité pour chacun des additifs, soit le brai B4. D’abord, l'anode 6 est fabriquée à partir du brai B4 non modifié afin de servir d'anode standard. Puis, quatre autres anodes ont été fabriquées afin d'étudier différents aspects. Puisque la concentration c4 de l'additif A3 a été étudiée avec le brai B1, cette même concentration est donc sélectionnée pour le brai B4 aux fins de comparaison. De plus, la concentration c2 de l'additif A3 a démontré une amélioration de la mouillabilité (Tableau 4) pour le brai B4 modifié contrairement au brai B1 modifié. Afin d'étudier l'effet de la variation de la concentration d'additif A3 sur le brai B4, les anodes 7 et 8 sont fabriquées à l'aide d'une concentration c2 et c4, respectivement. Pour l'additif A1, des concentrations de c1 et c3 sont sélectionnées afin de fabriquer les anodes 9 et 10. Ces mêmes concentrations ont été employées pour le brai B1. Le choix de ces concentrations d'additif A1 permet ainsi de comparer les résultats de la deuxième série d’anodes fabriquées à partir du brai B4 avec les résultats obtenus pour la première série d'anodes fabriquées à partir du brai B1. De plus, l'effet de l'augmentation de la quantité de l'additif A1 ajouté peut être également étudié. Le tableau 5 présente un sommaire des anodes produites et des brais employés dans la fabrication de ces anodes au laboratoire de carbone de l'UQAC. La recette d'agrégats secs est la même pour l'ensemble des anodes produites. 
Tableau 5. Sommaire des brais non modifiés et modifiés utilisés dans la fabrication des anodes.

\begin{tabular}{|c|c|c|c|}
\hline Anode & Brai & Additif & Concentration \\
\hline${\text { Anode } 1^{\mathrm{a}}}^{\mathrm{B}}$ & B1 & - & - \\
\hline Anode 2 $^{\mathrm{b}}$ & B1 & A1 & c3 \\
\hline Anode 3 & B1 & A1 & c3 \\
\hline Anode 4 & B1 & A1 & c1 \\
\hline Anode 5 & B1 & A3 & c4 \\
\hline Anode 6 & B4 & - & - \\
\hline Anode 7 & B4 & A3 & c2 \\
\hline Anode 8 & B4 & A3 & c4 \\
\hline Anode 9 & B4 & A1 & c1 \\
\hline Anode 10 & B4 & A1 & c3 \\
\hline
\end{tabular}

${ }^{\mathrm{a}}$ Anode standard produite avec les brais non modifiés

b Même composition que l'anode 3, mais produite en ajoutant de l'additif immédiatement avant la fabrication de l'anode (l'anode 3 est produite avec le brai pré-modifié).

\section{Caractérisation des brais modifiés}

Les groupements fonctionnels présents dans les brais B1 modifiés avec l'additif A1 préalablement et ceux recueillis après le préchauffage lors de la fabrication des anodes ont été analysés par spectroscopie infrarouge à transformée de Fourier (FTIR) à température ambiante. Les analyses sont réalisées avec le spectromètre Nicolet 6700 FTIR à raison de 26 balayages pour un nombre d'ondes compris entre 399 et $4000 \mathrm{~cm}^{-1}$. La résolution d'enregistrement a été fixée à 4 $\mathrm{cm}^{-1}$. La méthode d'analyse débute par le façonnage d'une pastille de bromure de potassium (KBr, grade FTIR) dont le spectre sert de blanc afin de diminuer l'effet de l'environnement sur l'analyse subséquente. Puis, chacun des brais modifiés est analysé à partir de pastilles de $\mathrm{KBr}$ contenant $1 \%$ d’analyte. Chaque expérimentation a été répétée trois fois en confectionnant une nouvelle pastille pour chacun des brais modifiés. L'ensemble des spectres IR enregistrés est transformé en spectre d'absorbance puis corrigé en effectuant une ligne de base tracée à partir du point le plus bas à $399 \mathrm{~cm}^{-1}$ jusqu'au point le plus bas à $4000 \mathrm{~cm}^{-1}$. Les spectres d'absorbance et les aires sous la courbe ont été obtenus à l'aide du logiciel Omnic 32 version 7,3. Enfin, l'absorbance des expérimentations et les aires sous la courbe ont été corrigées pour une concentration exacte de $0,01 \mathrm{~g} / \mathrm{g}$ d'analyte/ $\mathrm{KBr}$ et correspondent à la moyenne des trois essais. 


\section{Caractérisation des échantillons d'anodes}

Une fois que l'anode fabriquée est démoulée, celle-ci est pesée et mesurée afin de déterminer sa densité apparente crue. L’anode est ensuite carottée et coupée pour obtenir quatre échantillons crus puis séchés au four pendant 17 h selon le plan de la Figure 1 et de la Figure 2a. Chaque carotte est caractérisée afin d’obtenir leur densité apparente et leur résistance électrique spécifique selon les normes ASTM appropriées, respectivement D5502-00 et D6120-97.[16, 17] Les carottes 1 et 4 sont ensuite cuites puis caractérisées en mesurant leur densité apparente (ASTM D5502-00) et leur résistance électrique (ASTM D6120-97). ${ }^{[16,17]}$ Ensuite, la carotte 1 est employée pour tester sa résistance à la flexion en trois points (ISO CD 12986). ${ }^{[18]}$ Alors que la carotte 4, préalablement coupée selon le plan de la Figure 2b, est séchée 17 h avant d’être utilisée

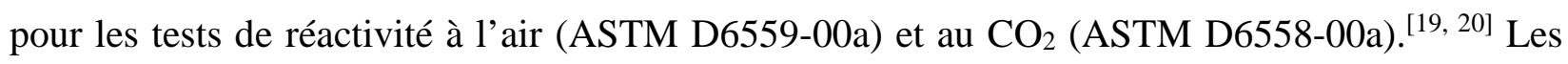
résultats de la caractérisation (densités et résistances électriques) constituent la moyenne des quatre échantillons crus ou la moyenne des deux échantillons cuits (carottes 1 et 4). Le Tableau 6 présente un plan des différents tests réalisés afin de caractériser les échantillons d’anodes.

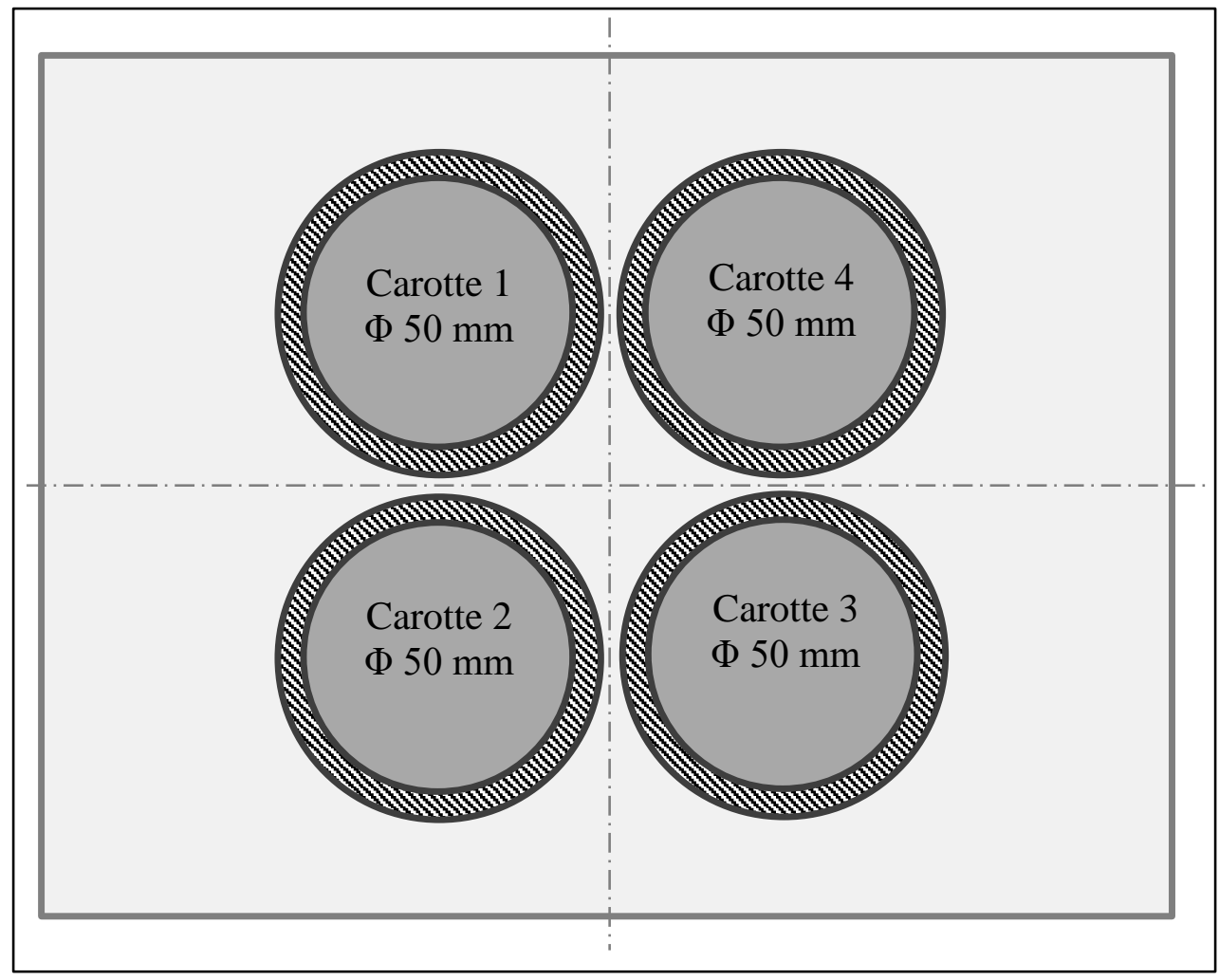

Figure 1. Plan de carottage des échantillons d’anodes crues vu de haut. 


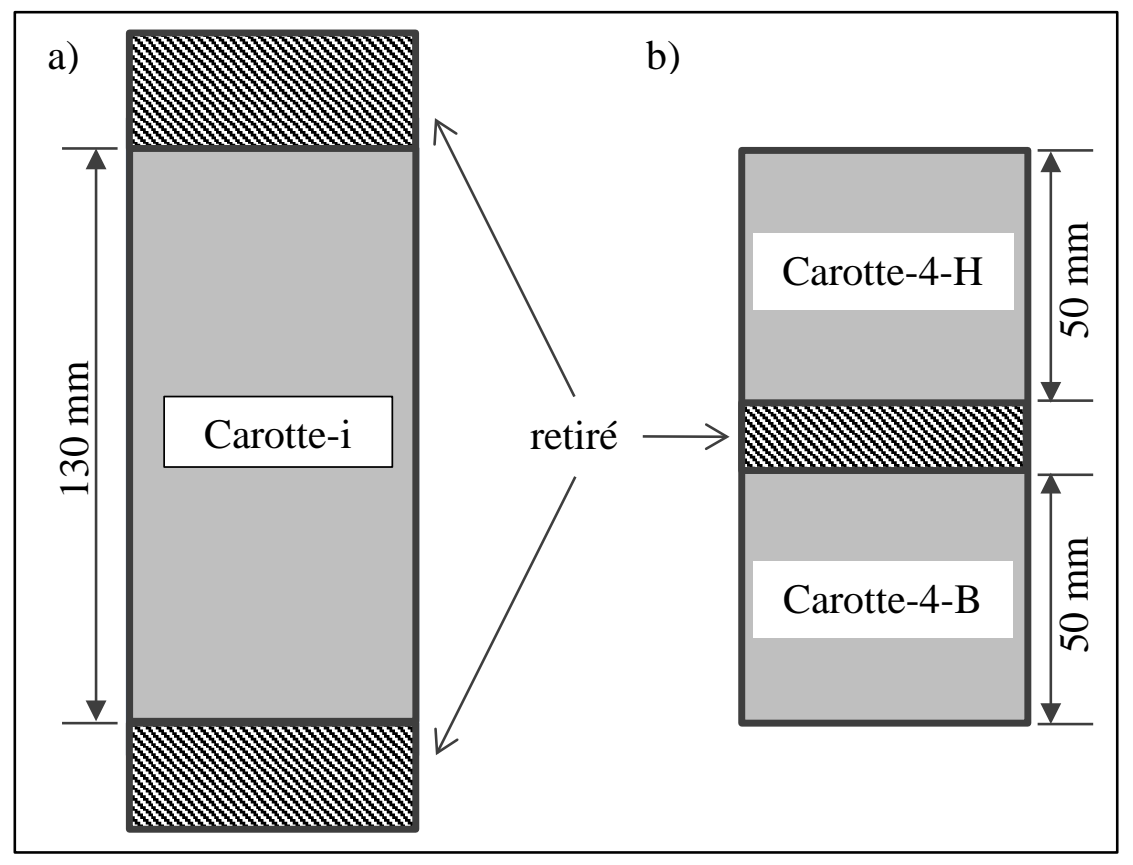

Figure 2. Plan de coupe de la carotte «i» de l’anode \# «i» vu de face

a) échantillons crues b) échantillon cuits du haut (H) et du bas (B).

Tableau 6. Plan de la caractérisation des échantillons

\begin{tabular}{|c|c|c|c|c|c|c|}
\hline \multirow{2}{*}{ Échantillon } & \multicolumn{2}{|c|}{ Cru } & \multicolumn{4}{c|}{ Cuit } \\
\hline & $\begin{array}{c}\text { Densité } \\
\text { apparente }\end{array}$ & $\begin{array}{c}\text { Résistivité } \\
\text { électrique }\end{array}$ & $\begin{array}{c}\text { Densité } \\
\text { apparente }\end{array}$ & $\begin{array}{c}\text { Résistivité } \\
\text { électrique }\end{array}$ & $\begin{array}{c}\text { Résistance à la } \\
\text { flexion }\end{array}$ & $\begin{array}{c}\text { Réactivité à } \\
\text { l'air et au } \mathrm{CO}_{2}\end{array}$ \\
\hline 1 & $\checkmark$ & $\checkmark$ & $\checkmark$ & $\checkmark$ & $\checkmark$ & \\
\hline 2 & $\checkmark$ & $\checkmark$ & & & & \\
\hline 3 & $\checkmark$ & $\checkmark$ & & & & \\
\hline 4 & $\checkmark$ & $\checkmark$ & $\checkmark$ & $\checkmark$ & & $\checkmark$ \\
\hline
\end{tabular}

\section{RÉSULTATS ET DISCUSSION}

\section{Analyse FTIR des brais modifiés}

Afin d'aider à sélectionner la méthode de modification, une analyse FTIR des brais modifiés A1c3B1 (additif A1, concentration c3, brai B1) des anodes 2 et 3 a été effectuée. Le brai nécessaire à la fabrication de l'anode 2 a été modifié en ajoutant l'additif lors de l'étape de préchauffage ( $1^{\text {ère }}$ méthode) immédiatement avant la fabrication de l'anode, tandis que le brai de l'anode 3 a été pré-modifié ( $2^{\mathrm{e}}$ méthode). La Figure 3 présente les spectres d'absorbance des brais 
recueillis après l'étape de préchauffage (identifié par «* ») lors de la fabrication des deux anodes (anode 2 et 3), de même que le brai préalablement modifié (identifié par «**») et utilisé plus tard pour la fabrication de l'anode 3. L'examen des spectres d'absorbances démontre plusieurs groupements fonctionnels contenus dans les brais analysés. La bande comprise entre 3000 et $3100 \mathrm{~cm}^{-1}$ peut être associée à une élongation de C-H aromatique. ${ }^{[21]}$ Tandis que les pics situés dans la région des 2800 et $2980 \mathrm{~cm}^{-1}$ représentent des liaisons C-H aliphatiques. ${ }^{[21]}$ Des pics situés entre 1660 et $1820 \mathrm{~cm}^{-1}$ correspondent au groupement fonctionnel d'un carbonyle $(\mathrm{C}=\mathrm{O}) .{ }^{[21]}$ La proximité des groupements voisins peut causer le déplacement de la position du pic associé au carbonyle. Pour ce motif, un nombre d'onde distinct de ce pic n'a pas été adopté pour l'analyse incluant donc tous les pics s'affichant entre 1660 et $1820 \mathrm{~cm}^{-1}$. Des pics associés aux alcanes $(C-C)$ ou alcènes $(C=C)$ sont observable dans la bande comprise entre 1400 et $1660 \mathrm{~cm}^{-}$ ${ }^{1.21]}$ Des groupements fonctionnels contenant des hétéroatomes (ester, éthers, alcool, phénols, amines, etc.) forment des pics dans la bande comprise entre 1000 et $1400 \mathrm{~cm}^{-1}{ }^{[21]}$ Finalement, les pics situés entre 700 et $900 \mathrm{~cm}^{-1}$ correspondent entre autres aux substitutions de l'anneau

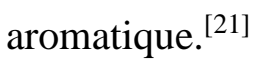

En observant les spectres de la Figure 3, il est possible de voir des différences significatives de l'absorbance de certains pics principaux entre 2800 et $2950 \mathrm{~cm}^{-1}$ ainsi qu'entre 1600 et $1750 \mathrm{~cm}^{-}$ ${ }^{1}$. Le Tableau 7 présente les résultats des aires sous la courbe de ces bandes d'intérêt. Il est possible de constater que les aires sous la courbe obtenue pour le brai préalablement modifié analysé avant la fabrication de l'anode possèdent des valeurs supérieures aux brais recueillis après l'étape de préchauffage. La diminution des valeurs indique une possible perte d'additif ou transformation de la composition chimique du brai au cours de l'étape de préchauffage. De plus, en observant l'aire sous la courbe pour les brais recueillis après l'étape de préchauffage, il est possible de constater un écart significatif. Les valeurs mesurées pour le brai modifié lors de l'étape de préchauffage (anode $2^{*}$ ) sont inférieures aux valeurs du brai ayant été préalablement modifié avant d'être préchauffé (anode $3 *$ ). La diminution de l'aire sous la courbe des pics obtenus pour l'anode $2 *$ signifie que la modification du brai au cours de l'étape de préchauffage n'est probablement pas aussi efficace que la modification préalable du brai (anode $3 *$ ). Cette constatation devra être soutenue par la caractérisation de ces deux anodes. 


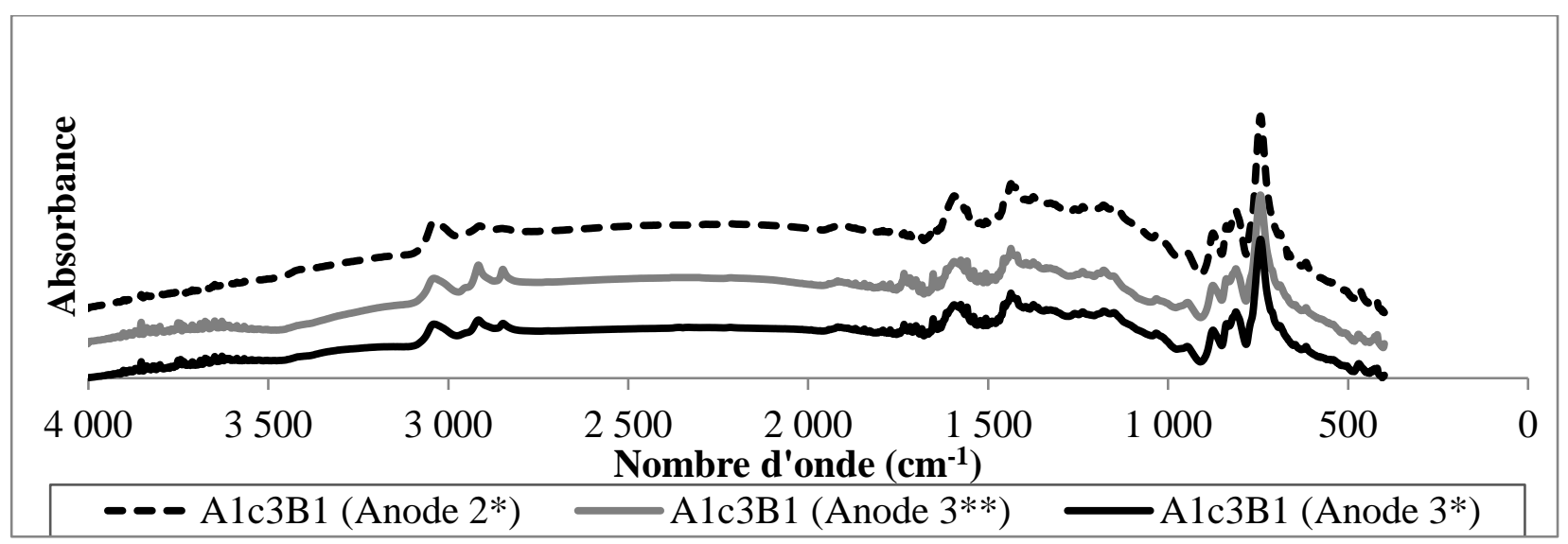

Figure 3. Spectre FTIR des brais B1 modifiés avec c3 de l'additif A1 pour la fabrication des anodes 2 et 3 .

\footnotetext{
* Brais recueillis après l'étape de préchauffage lors de la fabrication des anodes 2 et 3
}

**Brai préalablement modifié pour la fabrication de l'anode 3

Tableau 7. Aire sous la courbe des spectres FTIR du brai B1 modifié avec c3 de l'additif A1 pour la fabrication des anodes 2 et 3

\begin{tabular}{|c|c|c|c|}
\hline \multirow{2}{*}{$\begin{array}{c}\text { Bande comprise entre } \\
\left(\mathrm{cm}^{-1}\right)\end{array}$} & \multicolumn{3}{|c|}{ Aire sous la courbe du brai modifié A1c3B1 } \\
\cline { 2 - 4 } & Anode $3^{* *}$ & Anode $3^{*}$ & Anode $2^{*}$ \\
\hline 1660 et 1820 & 0,61 & 0,41 & 0,16 \\
\hline 2800 et 2980 & 1,00 & 0,65 & 0,38 \\
\hline
\end{tabular}

* Brais recueillis après l'étape de préchauffage lors de la fabrication des anodes 2 et 3

**Brai préalablement modifié pour la fabrication de l'anode 3

\section{Caractérisation des échantillons d'anodes}

Le Tableau 8 présente les résultats de la caractérisation de la première série d'anodes produites à partir du brai B1, tandis que le Tableau 9 présente les données recueillies pour la deuxième série d'anodes produites à partir du brai B4. Les données dans ces deux tableaux sont présentées sous la forme d'une différence entre la valeur pour l'anode actuelle et la valeur de l'anode standard. Une valeur positive montre que l'anode actuelle (fabriquée par le brai modifié) a une valeur plus élevée pour cette entité comparée à celle de l'anode standard et vice versa. Dès lors, les valeurs pour les anodes standards deviennent égales à zéro. Ces valeurs sont utilisées dans le calcul relatif à l'impact de l'ajout des additifs pour la production de l'aluminium dans la prochaine section.

Afin de favoriser la comparaison des résultats, les valeurs dans les tableaux 8 et 9 ont été normalisées entre -1 et +1 selon l'Équation 2 pour les anodes produites avec les brais modifiées. La valeur maximale est le plus grand écart (positif ou négatif) comparativement à la valeur 
standard. Les résultats des calculs sont présentés sous formes graphiques à la Figure 4, la Figure 5, la Figure 6 et la Figure 7. La Figure 4 et la Figure 5 présentent les résultats obtenus des anodes fabriquées à partir du brai B1, alors que la Figure 6 et la Figure 7 présentent les résultats de la caractérisation des anodes produites à partir du brai B4.

$$
\text { Valeur normalisée = Valeur à normaliser / | Valeur maximale | }
$$

Avant de procéder à la fabrication de l'ensemble des anodes, le choix de la méthode de modification du brai a été déterminé préalablement. En observant les anodes fabriquées (anodes 2 et 3) à partir du brai B1 modifié par l'additif A1 pour une concentration c3 (A1c3B1), il est possible d’observer un écart plus ou moins important entre les données (Tableau 8). Pour l'anode 2, l’additif A1 est ajouté au brai lorsque la température cible est atteinte. Une brève agitation est effectuée puis le mélange est ajouté rapidement au coke afin de ne pas abaisser la température de façon trop importante. Il est donc possible que le mélange ne soit pas uniforme. Ce résultat semble aussi être confirmé selon les résultats de la FTIR obtenus (Figure 3 et Tableau 7). Étant donné que les densités crues et cuites sont similaires, de même que le pourcentage de perte à la cuisson pour les anodes 2 et 3, une décomposition de l'additif lors de la fabrication est peu probable ou équivalente pour les deux méthodes. L’écart entre les absorbances signifie un manque possible d’homogénéité du mélange suggérant la présence de micelles. Par conséquent, le brai peut contenir par endroits une concentration supérieure en additif. Puisque l'additif A1 est un tensioactif, une concentration élevée en un endroit donné du brai est favorable à la création de micelles. La tête polaire est du tensioactif est donc disponible afin de se lier au brai, mais la queue hydrophobe est emprisonnée à l'intérieur de la micelle et donc inaccessible pour se lier au coke. Conséquemment, l'ensemble de l'additif ajouté directement pourrait procurer moins de sites actifs pour augmenter le nombre de liaisons possibles entre le brai et le coke. Dans le cas de la méthode de la modification préalable à la fabrication de l'anode, une agitation est effectuée jusqu’à ce que le mélange semble uniforme. Puisque les tensioactifs sont possiblement mieux répartis dans le mélange, la formation de micelles est moins favorisée. Par conséquent, les tensioactifs disponibles afin de se lier au Coke 1 dans l'anode 2 sont en quantité inférieure comparativement à l'anode 3. Donc, l'anode 2 renferme une concentration d'additif A1 probablement intermédiaire aux anodes 3 et 4 . La méthode de modification préalable du brai 
avant la fabrication des anodes a été employée pour les anodes suivantes afin de diminuer la possibilité de formation de micelle.

Une fois la méthodologie pour la modification du brai établie, la première série d'anodes a été complétée à partir du brai possiblement le moins compatible avec le Coke 1, soit le brai B1. En comparant les résultats des anodes produites avec le brai B1 non modifié et le brai B1 modifiés avec l'additif A1 (Tableau 8, Figure 4 et Figure 5), il est possible de remarquer une amélioration des densités et de la résistivité des carottes crues pour les anodes 2 et 3. Par contre, l'anode 4 (crue et cuite) présente des densités inférieures et des résistivités supérieures à l'anode standard. En effet, l'anode 4 produite à partir du brai B1 modifié par l'additif A1 pour une concentration c1, soit le brai modifié A1c1B1, possède une résistivité crue nettement supérieure aux trois autres anodes produites. Pourtant, la résistivité cuite de l'anode 4 ne s'écarte pas autant des valeurs mesurées pour les trois autres. La structure de cette anode renferme possiblement des fissures ou encore un manque de cohésion entre les particules. Concernant les tests de réactivité, aucune amélioration n'est obtenue pour la réactivité à l'air (sauf pour le poussiérage de l'anode 4) et certaines améliorations sont constatées pour la réactivité au $\mathrm{CO}_{2}$. En effet, le brai modifié A1c1B1 permet de maintenir la réactivité à l'air et de diminuer le poussiérage. Pour la réactivité au $\mathrm{CO}_{2}$, une amélioration est obtenue avec la diminution de la concentration de l'additif A1. De plus, l'ensemble des modifications avec l'additif A1 permet de diminuer le poussiérage en présence de $\mathrm{CO}_{2}$. De fait, il est difficile de réduire simultanément les réactivités de l'air et du $\mathrm{CO}_{2}$. Puisque la réactivité de l'air peut être contrôlée en améliorant la couverture des anodes. Il est donc préférable de réduire la réactivité au $\mathrm{CO}_{2}$. Enfin, seulement l'échantillon de l'anode 2 démontre de façon significative l'amélioration de la résistance à la flexion pour une concentration c3 de l'additif A1. Étant donné les résultats obtenus, il est possible d'affirmer que la modification du brai B1 par l'additif A1 peut améliorer certaines propriétés des anodes.

Pour la première série d'anodes produites, une seule anode a été fabriquée à partir de l'additif A3 pour une concentration c4 avec le brai B1, soit le brai modifié A3c4B1. Les données de la caractérisation démontrent une augmentation à la réactivité à l'air comparativement à l'anode standard. À l'inverse, une amélioration est observée pour la résistivité des carottes cuites, la résistance à la flexion, la densité de l'anode et des carottes crues, ainsi que la réactivité au $\mathrm{CO}_{2}$. Seules la résistivité pour les carottes crues et la densité des carottes cuites présentent des résultats 
similaires à l'anode standard. Conséquemment, ces résultats démontrent une amélioration significative des propriétés des anodes par la modification du brai B1 grâce à l'additif A3. Les résultats de la caractérisation ont permis d'obtenir une amélioration de certaines propriétés du brai $\mathrm{B} 1$ avec les additifs $\mathrm{A} 1$ et $\mathrm{A} 3$.

Tableau 8. Caractérisation des anodes fabriquées avec le Coke 1 et le brai B1

(moy. : moyenne, std : standard, Préfab : Préfabriqué, S.O. : sans objet)

\begin{tabular}{|c|c|c|c|c|c|c|}
\hline \multicolumn{2}{|c|}{ Anode } & 4 & 2 & 3 & $1(\mathrm{std})$ & 5 \\
\hline \multicolumn{2}{|c|}{ Méthode de modification } & Préfab. & Ajouté & Préfab. & S.O. & Préfab. \\
\hline \multicolumn{2}{|c|}{ Brai utilisé } & A1c1B1 & A1c3B1 & A1c3B1 & B1 & A3c4B1 \\
\hline \multirow{3}{*}{ Densité $\left(\mathrm{g} / \mathrm{cm}^{3}\right)$} & anode crue & $-0,036$ & 0,014 & 0,015 & 0 & 0,010 \\
\hline & carotte moy. crue & $-0,021$ & 0,03 & 0,024 & 0 & 0,019 \\
\hline & carotte moy. cuite & $-0,027$ & 0,006 & $-0,004$ & 0 & $-0,01$ \\
\hline \multicolumn{2}{|c|}{ \% de perte à la cuisson des carottes } & $-1,027$ & 0,606 & 0,535 & 0 & 0,476 \\
\hline \multirow{2}{*}{$\begin{array}{l}\text { Résistivité moyenne } \\
\text { des carottes }(\mu \Omega \cdot \mathrm{m})\end{array}$} & crue & 1779 & -486 & -640 & 0 & 151 \\
\hline & cuite & 1,56 & $-3,71$ & $-0,19$ & 0 & $-7,53$ \\
\hline \multirow{2}{*}{$\begin{array}{l}\text { Test de réactivité à } \\
\text { l'air des carottes } \\
\left(\mathrm{mg} /\left(\mathrm{cm}^{2} \cdot \mathrm{h}\right)\right)\end{array}$} & réactivité & 0,49 & 3,55 & 10,86 & 0 & 3,10 \\
\hline & poussiérage & $-0,157$ & 0,930 & 0,895 & 0 & 0,270 \\
\hline \multirow{2}{*}{$\begin{array}{l}\text { Test de réactivité au } \\
\mathrm{CO}_{2} \text { des carottes } \\
\left(\mathrm{mg} /\left(\mathrm{cm}^{2} \cdot \mathrm{h}\right)\right)\end{array}$} & réactivité & $-6,83$ & $-2,72$ & 0,61 & 0 & $-10,41$ \\
\hline & poussiérage & $-1,909$ & $-1,506$ & $-3,224$ & 0 & $-3,788$ \\
\hline \multicolumn{2}{|c|}{ Test de résistance à la flexion (MPa) } & $-0,447$ & 1,683 & 0,180 & 0 & 2,767 \\
\hline
\end{tabular}




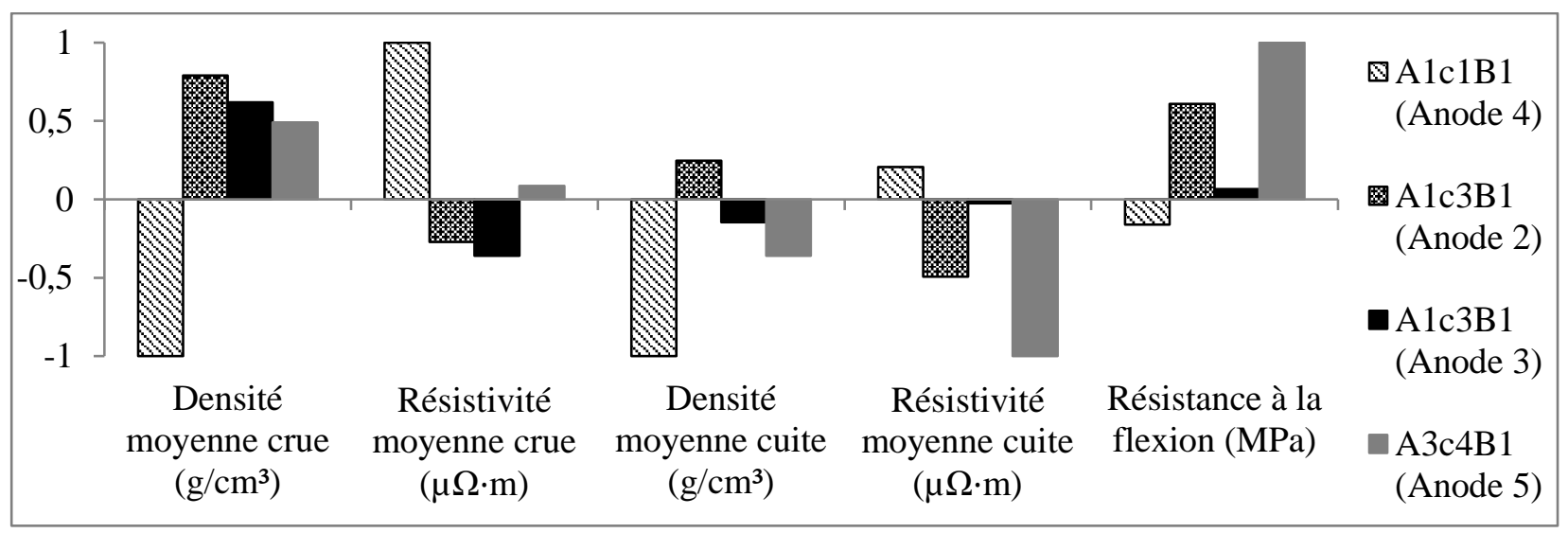

Figure 4. Comparaison des propriétés physiques des anodes fabriquées avec le Coke 1 et le brai B1.

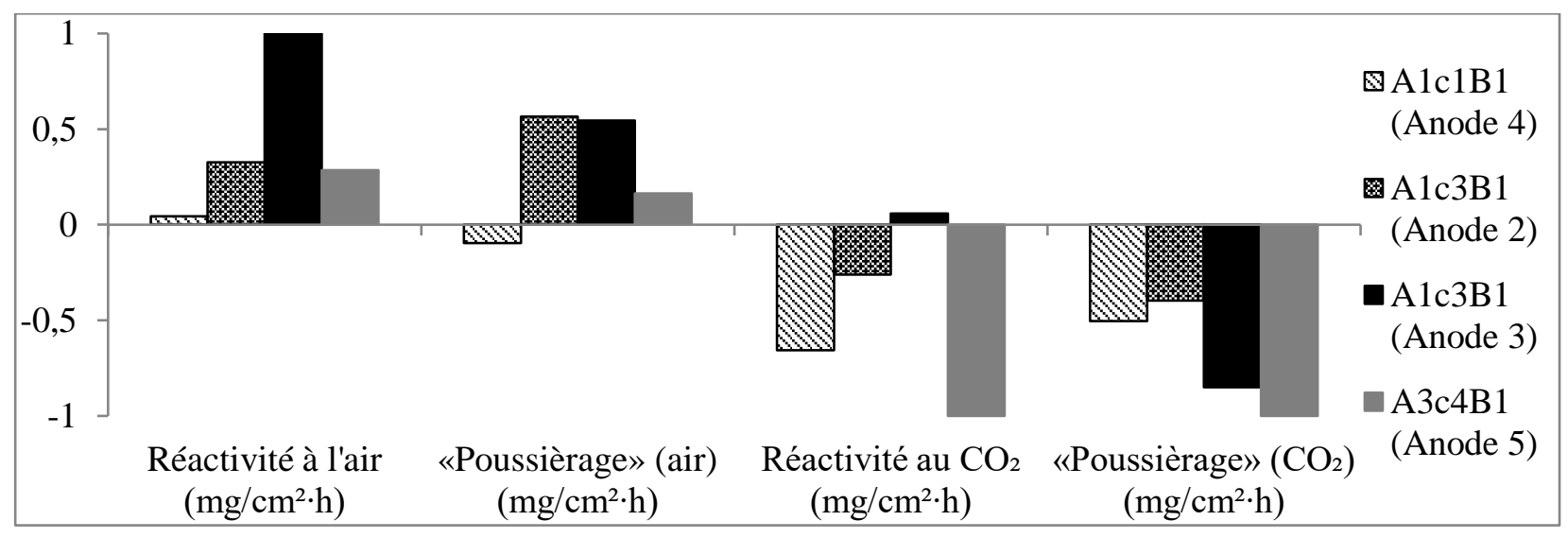

Figure 5. Comparaison de la réactivité à l'air et au $\mathrm{CO}_{2}$ des anodes fabriquées avec le Coke 1 et le brai B1.

Selon les résultats obtenus dans une étude antérieure, le brai B4 non modifié a démontré un temps de mouillage total inférieur au brai B1. ${ }^{[15]}$ Par conséquent, le brai B4 non modifié est probablement plus compatible avec le Coke 1 que le brai B1 non modifié. Afin d'étudier l'effet de l'ajout des additifs A1 et A3 sur un brai plus compatible au Coke 1 que le brai B1, une deuxième série d’anodes a été fabriquée avec le brai B4. Une amélioration des propriétés des anodes avec le brai B4 modifié signifie que la modification du brai par l'additif pour une concentration donnée est probablement aussi efficace pour des brais de compatibilités intermédiaires au brai B1 et B4. 
Les résultats des échantillons d'anode fabriquée avec le brai B4 non modifié ont été comparés à celles modifiées avec les additifs A1 et A3 (Tableau 9, Figure 6 et Figure 7). En observant les données à la Figure 6, il est possible de remarquer une diminution des densités pour l'ensemble des anodes fabriquées à partir de brai modifié comparativement à l'anode standard, exceptée pour les anodes 8 et 10 crues qui présentent une certaine amélioration. Concernant la résistivité des échantillons crus et cuits des anodes faites de brai modifié, les résultats montrent une augmentation des valeurs pour l'ensemble des anodes comparativement à l'anode standard, excepté l'anode 8 cuite dont la valeur a diminué. Enfin, l'ensemble des brais modifiés a permis d'améliorer la résistance à la flexion des anodes produites comparativement à l'anode standard, excepté pour l'anode 10 dont la résistance à la flexion a diminué. Ces résultats démontrent que la modification du brai par l'additif A1 ou l'additif A3 ne semble pas améliorer les propriétés physiques de l'anode comparativement à l'anode standard dont le brai non modifié contenu est déjà favorable à l'interaction coke/brai. Selon les résultats obtenus par test goutte-sessile (Tableau 4), la modification du brai B4 diminue le temps de mouillage total. Une amélioration de la mouillabilité signifie une possible amélioration de la compatibilité du brai avec le coke. Pourtant, les résultats de la caractérisation des anodes ne permettent pas de démontrer une amélioration de l'interaction entre le brai B4 modifié et le Coke 1. Une explication possible de ce résultat est que l'amélioration de la mouillabilité par la modification du brai B4 a davantage amélioré sa compatibilité avec le Coke 1 comparativement au brai B4 non modifié, de sorte que la quantité de brai modifié nécessaire pour assurer la cohésion entre les agrégats secs est supérieure à l'optimal. Il en résulte une diminution de la densité et la libération possible de davantage de volatiles causant des fissures, ce qui entraîne une augmentation de la résistivité.

La Figure 7 présente les résultats des tests de réactivité des échantillons d'anodes cuites faites à partir du brai B4 modifié avec l'additif A1 ou A3. Il est possible de constater une diminution de la réactivité à l'air et une augmentation du poussiérage à l'air pour l'ensemble des anodes produites (sauf l'anode 10) comparativement à l'anode standard. En effet, l'anode 10 faite à partir d'une concentration c3 de l'additif A1 augmente la réactivité à l'air comparativement à l'anode standard. Pour les résultats de la réactivité au $\mathrm{CO}_{2}$, il n’y a aucune différence significative pour les anodes 7 et 10, une augmentation de la réactivité pour l'anode 9 et une diminution de la réactivité pour l'anode 8. Pour ce qui est du poussiérage associé, aucune différence significative n'est obtenue pour l'anode 10, une augmentation est observée pour les anodes 7 et 9 , tandis 
qu'une diminution est constatée pour l'anode 8. Il est donc possible d'affirmer que les résultats obtenus pour les anodes fabriquées à partir du brai B4 modifié avec l'additif A1 ne permettent pas de démontrer une amélioration significative lorsque le brai non modifié est déjà favorable sauf pour la réactivité à l'air pour une concentration c1. En ce qui concerne l'additif A3, de façon générale, l'augmentation de la concentration de l'additif A3 pour modifier le brai B4 diminue les réactivités à l'air et au $\mathrm{CO}_{2}$ ainsi que le poussiérage associé. De plus, une concentration de c4 de l'additif A3 permet d'obtenir de meilleurs résultats comparativement à l'anode standard sauf pour le poussiérage mesuré lors des tests de réactivité à l'air.

Tableau 9. Caractérisation des anodes fabriquées avec le Coke 1 et le brai B4 (moy. : moyenne, std : standard, Préfab : Préfabriqué, S.O. : sans objet)

\begin{tabular}{|c|c|c|c|c|c|c|}
\hline \multicolumn{2}{|c|}{ Anode } & 7 & 8 & 6 (std) & 9 & 10 \\
\hline \multicolumn{2}{|c|}{ Méthode de modification } & Préfab. & Préfab. & S.O. & Préfab. & Préfab. \\
\hline \multicolumn{2}{|c|}{ Brai utilisé } & A3c2B4 & A3c4B4 & B4 & A1c1B4 & A1c3B4 \\
\hline \multirow{3}{*}{ Densité $\left(\mathrm{g} / \mathrm{cm}^{3}\right)$} & anode crue & $-0,006$ & 0,004 & 0 & $-0,013$ & 0,012 \\
\hline & carotte moy. crue & $-0,012$ & $-0,001$ & 0 & $-0,017$ & 0,003 \\
\hline & carotte moy. cuite & $-0,025$ & $-0,008$ & 0 & $-0,018$ & $-0,009$ \\
\hline \multicolumn{2}{|c|}{ \% de perte à la cuisson des carottes } & 0,382 & 0,477 & 0 & $-0,138$ & 0,633 \\
\hline \multirow{2}{*}{$\begin{array}{c}\text { Résistivité moy. } \\
\text { des carottes }(\mu \Omega \cdot \mathrm{m})\end{array}$} & crue & 552 & 124 & 0 & 656 & 419 \\
\hline & cuite & 2,98 & $-1,54$ & 0 & 3,73 & 0,86 \\
\hline \multirow{2}{*}{$\begin{array}{c}\text { Test de réactivité à } \\
\text { l’air des carottes } \\
\left(\mathrm{mg} /\left(\mathrm{cm}^{2} \cdot \mathrm{h}\right)\right)\end{array}$} & réactivité & $-3,05$ & -6.16 & 0 & $-10,26$ & 3,87 \\
\hline & poussiérage & 1,64 & 1,02 & 0 & 0,17 & 1,13 \\
\hline \multirow{2}{*}{$\begin{array}{c}\text { Test de réactivité au } \\
\mathrm{CO}_{2} \text { des carottes } \\
\left(\mathrm{mg} /\left(\mathrm{cm}^{2} \cdot \mathrm{h}\right)\right)\end{array}$} & réactivité & 0,13 & $-2,91$ & 0 & 1,32 & $-0,20$ \\
\hline & poussiérage & 0,84 & $-1,75$ & 0 & 0,78 & 0,02 \\
\hline \multicolumn{2}{|c|}{ Test de résistance à la flexion (MPa) } & 0,415 & 1,126 & 0 & 1,124 & $-0,407$ \\
\hline
\end{tabular}




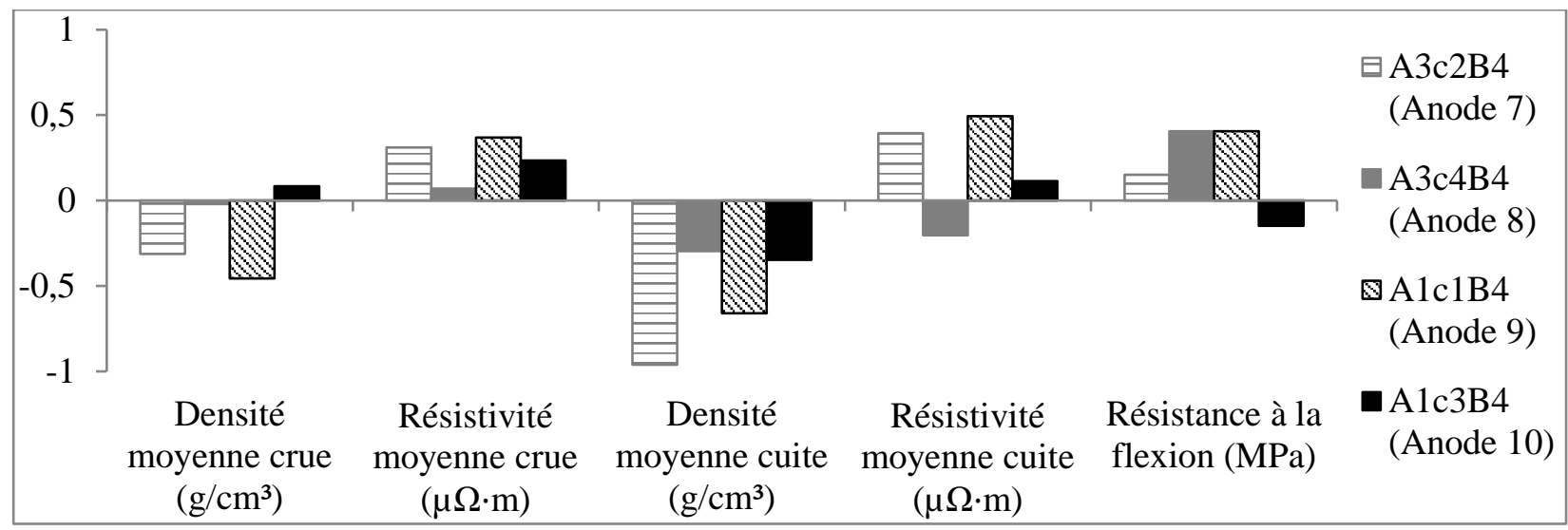

Figure 6. Comparaison de la caractérisation physique des anodes fabriquées avec le Coke 1 et le brai B4.

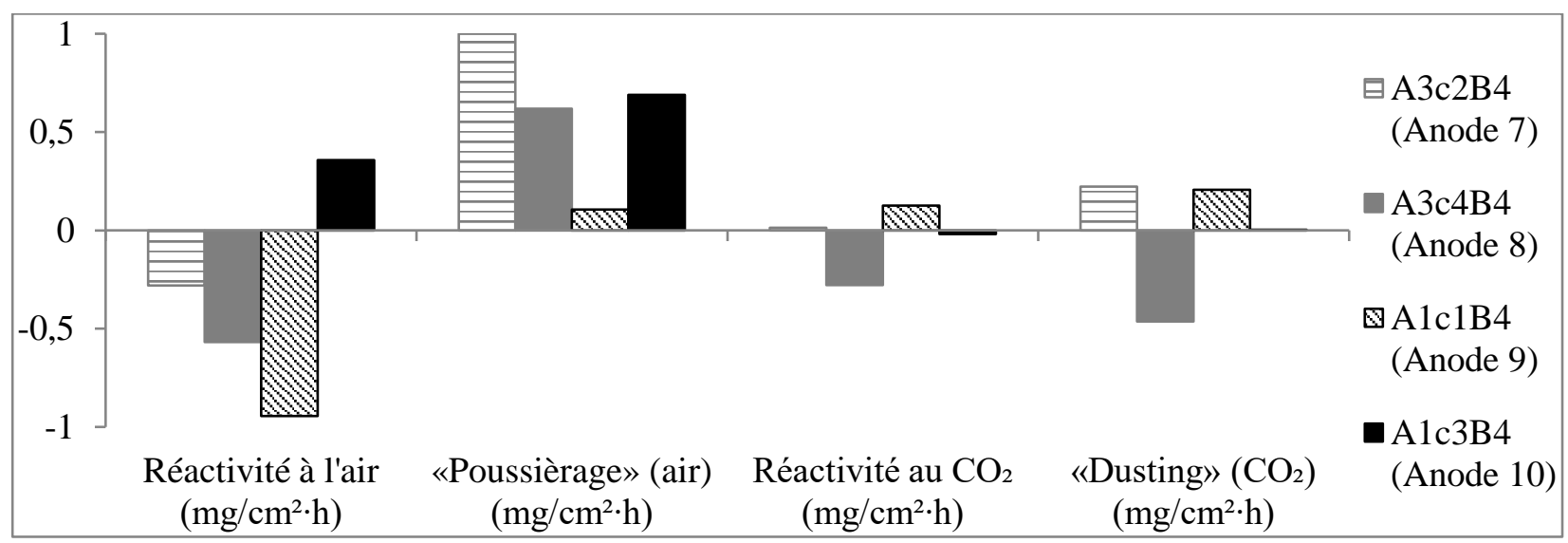

Figure 7. Comparaison de la réactivité à l'air et au $\mathrm{CO}_{2}$ des anodes fabriquées avec le Coke 1 et le brai B4.

Dans le but de mieux comprendre l'ensemble des résultats de la caractérisation des anodes, une étude de l’impact économique et sur la production de l'aluminium a été réalisée et présentée à la section suivante.

\section{Impacts possibles de l'ajout des additifs chimiques}

Étant donné que la production d'une liaison satisfaisante entre les agrégats secs et le brai affectent fortement le procédé électrolytique, une amélioration significative des propriétés des anodes peut avoir un impact considérable sur le coût de production du métal. Par exemple, sachant qu'une anode de 0,6 m (L) avec une surface inférieure de $1 \mathrm{~m}^{2}$ (A) et que l'intensité (I) qui passe par une anode est d'environ $10 \mathrm{kA}$ (soit $400 \mathrm{kA}$ par 40 anodes), alors une amélioration 
de la résistivité spécifique ( $\rho$ ) de $1 \mu \Omega \cdot m$ correspond à une amélioration de la résistance $(R)$ de $0,6 \mu \Omega$ selon l'équation 3 :

$$
\rho(\mathrm{L} / \mathrm{A})=1 \mu \Omega \cdot \mathrm{m}\left(0,6 \mathrm{~m} / 1 \mathrm{~m}^{2}\right)=0,6 \mu \Omega
$$

Ainsi, une amélioration de la résistivité de $1 \mu \Omega \cdot \mathrm{m}$, pour un courant moyen de $400 \mathrm{kA}$, diminue la puissance $(\mathrm{P})$ de production requise de $60 \mathrm{~W}$ selon l'équation 4 :

$$
\mathrm{P}=\mathrm{I}^{2} \cdot \mathrm{R}=(10000 \mathrm{~A})^{2}\left(0,6 \times 10^{-6} \Omega\right)=60 \mathrm{~W}
$$

Ce qui correspond à une économie d'environ $30 \mathrm{kWh}$ sur la durée de vie moyenne de 21 jours d'une anode. ${ }^{[2]}$ De plus, en se basant sur une densité moyenne (D) de l'anode cuite de 1,55 $\mathrm{kg} / \mathrm{dm}^{3}$, un gain de $0,01 \mathrm{~kg} / \mathrm{dm}^{3}$ en densité $(\Delta \mathrm{D})$ représente environ 6 à $7 \mathrm{~kg}$ de carbone supplémentaire ( $\left.\mathrm{m}_{\text {carbone}}\right)$ par anode de $1000 \mathrm{~kg}$ ( $\left.\mathrm{m}_{\text {anode }}\right)$ selon l'équation $5:^{[1,2]}$

$$
\mathrm{m}_{\text {carbone }}=\Delta \mathrm{D} \cdot \mathrm{m}_{\text {anode }} / \mathrm{D}=0,01 \mathrm{~kg} / \mathrm{dm}^{3} \cdot 1000 \mathrm{~kg} / 1,55 \mathrm{~kg} / \mathrm{dm}^{3} \approx 6,5 \mathrm{~kg}
$$

Enfin, une diminution de la réactivité au $\mathrm{CO}_{2}$ ou à l'air (Réact.) de $1 \mathrm{mg} / \mathrm{cm}^{2} \cdot \mathrm{h}$ représente une économie d'environ $5 \mathrm{~kg}$ de carbone par cycle de vie d'une anode par réactivité (soit un temps $\mathrm{t}=$ 504 h) selon l'équation 6 :

$$
\mathrm{m}_{\text {carbone }}=\text { Réact. } \cdot \mathrm{A} \cdot \mathrm{t}=1 \mathrm{mg} / \mathrm{cm}^{2} \cdot \mathrm{h} \cdot 10000 \mathrm{~cm}^{2} \cdot 504 \mathrm{~h} \approx 5 \mathrm{~kg} \text { de carbone }
$$

Considérant que l'équation 6 est calculée à partir de l'aire à la base de l'anode, sachant qu'une portion des côté de l'anode réagit elle aussi, l'économie en carbone obtenue est probablement supérieure à $5 \mathrm{~kg}$. Les gains en carbone réalisés procurent une production supplémentaire d'environ 20-25 kg d'aluminium par anode en considérant la densité et la réactivité totale d'une anode.

Le Tableau 10 et le Tableau 11 présentent les impacts possibles de la modification du brai sur la consommation et la production comparativement aux valeurs moyennes obtenues en industrie pour l'ensemble des anodes produites contenant du brai modifié. Les résultats présentés ont été calculés à partir des données présentées au Tableau 8 et au Tableau 9 selon les informations citées au paragraphe précédent. Bien que les résultats de la caractérisation n’aient pas démontré une augmentation significative de la densité spécifique apparente, il est tout de même possible d'observer une diminution en carbone consommé pour certaines modifications du brai. Ce gain 
s'explique grâce à la diminution de la réactivité à l'air et/ou au $\mathrm{CO}_{2}$ comparativement à l'anode standard. Pour le brai ayant la moins bonne compatibilité, le brai B1, certaines améliorations sont observées. L'additif A1 permet une économie en carbone et une augmentation de la production en aluminium pour une concentration c1 (anode 4) et une économie en énergie consommée pour l'anode 2. Tandis que l'additif A3, ajouté selon une concentration c4, permet une économie énergétique et en carbone consommé tout en augmentant la production d'aluminium. En ce qui a trait au brai ayant la meilleure compatibilité étudiée, le brai B4, certaines améliorations sont aussi obtenues. Pour l'additif A1, il y a diminution de la consommation en carbone causé par la diminution de la réactivité à l'air et par conséquent une augmentation de la production en aluminium pour une concentration de c1 (anode 9). Toutefois, considérant l'augmentation énergétique due à l'augmentation de la résistivité, la modification du brai B4 par l'ajout d'une concentration c1 de l'additif A1 n'est probablement pas bénéfique globalement pour la quantité de brai modifié utilisée puisque l'interaction brai B4/Coke 1 est déjà satisfaisante. En ce qui concerne l'additif A3, l'augmentation de la concentration pour modifier le brai B4 permet une diminution de la quantité de carbone consommé et une augmentation de la production en aluminium (anode 7 et 8). De plus, une concentration c4 procure également une économie énergétique (anode 8).

Tableau 10. Impacts possibles de la modification du brai B1sur la consommation et la production comparativement à l'anode standard, basés sur une anode de 1000 kg et un courant de 400 kA (les valeurs sans parenthèse indiquent un impact positif et celles entre parenthèses un impact négatif).

\begin{tabular}{|c|c|c|c|c|c|}
\hline Anode & 4 & 2 & 3 & $1(\mathrm{std})$ & 5 \\
\hline Brai utilisé & A1c1B1 & A1c3B1 & A1c3B1 & B1 & A3c4B1 \\
\hline Énergie économisée $(\mathrm{kWh})$ & $(52)$ & 124 & 6 & 0 & 253 \\
\hline Quantité de carbone économisé $(\mathrm{kg})$ & 31 & $(4)$ & $(56)$ & 0 & 36 \\
\hline Aluminium supplémentaire produit $(\mathrm{kg})$ & 77 & $(10)$ & $(139)$ & 0 & 89 \\
\hline
\end{tabular}


Tableau 11. Impacts possibles de la modification du brai B4 sur la consommation et la production comparativement à l'anode standard, basés sur une anode de $1000 \mathrm{~kg}$ et un courant de $400 \mathrm{kA}$ (les valeurs sans parenthèse indiquent un impact positif et celles entre parenthèses un impact négatif).

\begin{tabular}{|c|c|c|c|c|c|}
\hline Anode & 7 & 8 & 6 (std) & 9 & 10 \\
\hline Brai utilisé & A3c2B4 & A3c4B4 & B4 & A1c1B4 & A1c3B4 \\
\hline Énergie économisée $(\mathrm{kWh})$ & $(100)$ & 51 & 0 & $(125)$ & $(29)$ \\
\hline Quantité de carbone économisé $(\mathrm{kg})$ & 14 & 45 & 0 & 44 & $(18)$ \\
\hline Aluminium supplémentaire produit $(\mathrm{kg})$ & 35 & 110 & 0 & 108 & $(45)$ \\
\hline
\end{tabular}

\section{CONCLUSIONS}

Des brais modifiés prometteurs avaient été sélectionnés dans une étude antérieure basés sur l'amélioration de la mouillabilité du coke par ces brais. ${ }^{[15]}$ Dans ce projet, l'effet de l'utilisation d'un brai modifié chimiquement pour produire des anodes de laboratoire a été étudié. Des anodes de laboratoire ont été fabriquées à partir d'un brai moins compatible avec un coke standard ainsi qu'avec un brai plus compatible.

L'analyse des résultats permet d'affirmer que l'usage d'un additif peut procurer une amélioration de certaines propriétés des anodes. Plus précisément, la modification par l'additif A1, à faible concentration, permet d'améliorer certaines des propriétés du brai, quand l'interaction coke-brai non modifié n’est pas favorable (brai B1). Si cette interaction est déjà favorable (brai B4), l'additif A1 a peu d'impact. La modification par l'additif A3 semble prometteuse, surtout pour une concentration supérieure à c2. Cet additif a permis d'améliorer les propriétés des anodes fabriquées avec les brais B1 et B4 en augmentant la résistance à la flexion et en diminuant la résistivité électrique ainsi que les réactivités à l'air et/ou au $\mathrm{CO}_{2}$ comparativement à l'anode standard correspondante. La diminution de la densité apparente spécifique comparativement à l'anode standard associée laisse supposer que l'anode fabriquée avec le brai modifié par l'additif A3 est en sur-brai. Dans cette étude, toutes les anodes ont été faites en utilisant la même quantité de brai. Considérant la modification des propriétés du brai due à l'usage des additifs, il est 
possible que la quantité optimale de brai requise puisse changer. Donc, il est possible qu'une amélioration supplémentaire des propriétés de l'anode soit obtenue en ajustant le niveau de brai.

\section{REMERCIEMENTS}

Nous tenons à remercier vivement le soutien du Conseil de recherches en sciences naturelles et en génie du Canada (CRSNG), de l'Université du Québec à Chicoutimi (UQAC) et de la Fondation de l'Université du Québec à Chicoutimi (FUQAC).

\section{RÉFÉRENCES}

1. Hulse K. L., Anode manufacture : raw materials, formulation and processing parameters, R \& D Carbon Ltd., Sierre, Suisse 2000, p. xxxv.

2. A. Charette, Y. S. Kocaefe, D. Kocaefe, Le carbone dans l'industrie de l'aluminium. xxi ed, Les presses de l'aluminium, Chicoutimi, Québec 2012, p. 325.

3. A. D. Kamran, Investigation of the materials and paste relationships to improve forming process and anode quality, Thèse de doctorat, Université Laval, Québec, Canada 2013, p. 148.

4. A. Sarkar, D. Kocaefe, Y. S. Kocaefe, D. Sarkar, D. Bhattacharyay, B. Morais, J. Chabot, Fuel. Coke-pitch interactions during anode preparation. 2014, 117 (PART A), 598.

5. S. M. Hume, Anode reactivity : influence of raw material properties. [2nd ed.]. ed, R \& D Carbon Ltd., Sierre [Suisse] 1999, p. xi.

6. Y. Lu, Effect of pitch properties on anode properties, Mémoire de maîtrise, Université du Québec à Chicoutimi, Chicoutimi, Québec, Canada 2016, p. 221.

7. S. M. Oh, Y. D. Park, Fuel. Comparative studies of the modification of coal-tar pitch. 1999, 78 (15), 1859.

8. V. G. Rocha, M. Granda, R. Santamaría, C. Blanco, E. I. Diestre, R. Menéndez, «Improvement of pitch/coke interactions by addition of surface tension modifiers», Carbon2004, Brown University, Providence, RI, 11-16 July 2004.

9. V. G. Rocha, C. Blanco, R. Santamaría, E. I. Diestre, R. Menéndez, M. Granda, Fuel. Pitch/coke wetting behaviour. 2005, 84 (12), 1550.

10. Q. Lin, T. Li, C. Zheng, Y. Zhao, S. Song, Journal of Analytical and Applied Pyrolysis. Carbonization behavior of coal-tar pitch modified with divinylbenzene and optical texture of resultant semi-cokes. 2004, 71 (2), 817.

11. Q. Lin, T. Li, Y. Ji, W. Wang, X. Wang, Fuel. Study of the modification of coal-tar pitch with p-methyl benzaldehyde. 2005, 84 (2-3), 177.

12. V. G. Rocha, C. Blanco, R. Santamaría, E. I. Menéndez, R. Granda, M. Diestre, Fuel Processing Technology. The effect of the substrate on pitch wetting behaviour. 2010, 91 (11), 1373.

13. European Commission, The use of coal-tar pitches of very high softening point and low carcinogen content as binders for industrial carbon, in Technical Coal Research, Report No. EUR 18584 EN. 1999: Office for Publication of the Europenne Communities, Luxemburg. p. 48.

14. Rocha V. G., Granda M., Santamaría R., Blanco C., Diestre E. I., Menéndez R., Journal of Analytical and Applied Pyrolysis. Pyrolysis behaviour of pitches modified with different additives. 2005, 73 (2), 276. 
15. J. Bureau, D. Kocaefe, Y. Kocaefe, D. Bhattacharyay, B. Morais, The Canadian Journal of Chemical Engeineering. Modification des propriétés du brai de goudron de houille utilisé dans la fabrication des anodes. 2017.

16. An American National Standard, Standard test method for electrical resistivity of anode and cathode carbon material at room temperature, in ASTM D6120-97 (Réapprovée en 2007). 2010. p. 3 pages.

17. An American National Standard, Standard test method for apparent density by physical measurements of manufactured anode and cathode carbon used by the aluminum industry, in ASTM D5502-00 (Réapprouvé en 2005). 2010. p. 3 pages.

18. International Standard, Carbonaceous materials used in the production of aluminium -Prebaked anodes and cathode blocks -- Part 1: Determination of bending/shear strength by a three-point method, in ISO 12986-1:2000. 2000. p. 7 pages.

19. An American National Standard, Standard test method for determination of tga air reactivity of baked carbon anodes and cathode blocks, in ASTM D6559-00a (Réapprouvé en 2005). 2010. p. 5 pages.

20. An American National Standard, Standard test method for determination of tga $\mathrm{CO}_{2}$ reactivity of baked carbon anodes and cathode blocks, in ASTM D6558-00a (réapprouvée en 2005). 2010. p. 5 pages.

21. N. B. Colthup, J. Opt. Soc. Am. Spectra-Structure Correlations in the Infra-Red Region. 1950, 40 (6), 397. 


\section{NOMENCLATURE}

BGH Brai de goudron de houille

CMCNa Carboxyméthyl-cellulose de sodium

M40 Mobilsol 40

PS Polystyrène

ci Concentration (où i $=1,2,3$ ou 4$)(\% \mathrm{~g} / \mathrm{g})$

$\mathrm{KBr} \quad$ Bromure de potassium

FTIR Spectroscopie infrarouge à transformée de Fourier

Bi $\quad$ Brai 1 (où $i=1$ ou 4 )

Ai $\quad$ Additif (où $i=1$ ou 4 ) 